\title{
Quantification of SARS-CoV-2 antigen levels in the blood of patients with COVID-19
}

\author{
Bin $\mathrm{Su}^{1 \dagger}$, Jiming $\mathrm{Yin}^{1 \dagger}{ }^{1 \dagger}$, Xingguang $\mathrm{Lin}^{2}$, Tiantian Zhang ${ }^{3}$, Xiao Yao ${ }^{4}$, Ying $\mathrm{Xu}^{4}$, Yao Lu ${ }^{5}$, \\ Wenzhi Wang ${ }^{5,6}$, Kun Liu ${ }^{2}$, Jie Zhang ${ }^{7}$, Liangzhi Xie ${ }^{7,8,9^{*}}$, Ronghua Jin $^{1^{*}}$ \& Yingmei Feng ${ }^{1^{*}}$ \\ ${ }^{1}$ Beijing Youan Hospital, Capital Medical University, Beijing 100069, China; \\ ${ }^{2}$ Hangzhou Gbio Biotechnology Co., Ltd., Hangzhou 310000, China; \\ ${ }^{3}$ Hangzhou Neoline Technology Co., Ltd., Hangzhou 310000, China; \\ ${ }^{4}$ Quanterix Corporation, 900 Middlesex Turnpike, Billerica, MA 01821, USA; \\ ${ }^{5}$ Guizhou Academy of Testing and Analysis, Guiyang 550014, China; \\ ${ }^{6}$ Wildlife Forensic Science Service, Kunming 650203, China; \\ ${ }^{7}$ Beijing Key Laboratory of Monoclonal Antibody Research and Development, Sino Biological Inc. Beijing 100176, China; \\ ${ }^{8}$ Beijing Engineering Research Center of Protein and Antibody, Sinocelltech Ltd., Beijing 100176, China; \\ ${ }^{9}$ Cell Culture Engineering Center, Chinese Academy of Medical Sciences \& Peking Union Medical College, Beijing 100005, China
}

Received August 18, 2020; accepted November 11, 2020; published online November 26, 2020

Citation: Su, B., Yin, J., Lin, X., Zhang, T., Yao, X., Xu, Y., Lu, Y., Wang, W., Liu, K., Zhang, J., Xie, L., Jin, R., and Feng, Y. (2021). Quantification of SARSCoV-2 antigen levels in the blood of patients with COVID-19. Sci China Life Sci 64, 1193-1196. https://doi.org/10.1007/s11427-020-1830-8

\section{Dear Editor,}

Severe acute respiratory syndrome coronavirus 2 (SARSCoV-2) has caused the global outbreak of coronavirus disease 2019 (COVID-19). By far, more than 35 million people had been infected by SARS-CoV-2, resulting in more than 1 million deaths globally. It is well recognized that SARSCoV-2 preferentially attacks pulmonary epithelial cells, leading to acute respiratory distress syndrome (ARDS). Remarkably, infected patients experience liver (Phipps et al., 2020), kidney (Gabarre et al., 2020) or heart injury (Bavishi et al., 2020), indicating the presence of multiple-organ dysfunction via viremia.

By far, viral detection in RNA extracted from nasopharyngeal swabs, saliva, sputum, and bronchoalveolar lavage fluid has remained the standard etiological diagnostic procedure for COVID-19, with the detection rate ranging from $70 \%$ to more than $90 \%$ (Lippi et al., 2020). Paradoxically, the

$\dagger$ Contributed equally to this work

*Corresponding authors (Yingmei Feng, email: yingmeif13@sina.com; Ronghua Jin, email: jin_eagle@sina.com; Liangzhi Xie, email: liangzhi@yahoo.com) viral RNA detection rate is only $15 \%-30 \%$ in blood samples (Scohy et al., 2020; Mak et al., 2020). Moreover, hampered by the low performance of the current technology, few studies have evaluated SARS-CoV-2 antigen levels in the blood. The single molecular array $\left(\right.$ SIMOA $\left.^{\mathrm{TM}}\right)$ ultrasensitive platform employs enzyme-conjugated microscopic beads for protein capture and enables antigen quantification at femtomolar concentrations (Rissin et al., 2010). In the study, we investigated whether the SIMOA method could determine SARS-CoV-2 antigen levels in patients with COVID-19.

The general characteristics of the patients enrolled in the study are presented in Table S1 in Supporting Information. The patients were aged 57.9 years. Among 35 patients, 15 $(42.9 \%)$ were males, $11(31.4 \%)$ had histories of staying in or traveling to Wuhan in 14 days before diagnosis, and 10 $(28.6 \%)$ were severe or critically ill. Of the 10 severe or critically ill patients, five experienced ARDS.

Assays measuring human plasma COVID-19 nucleocapsid protein ( $\mathrm{N}$ protein) expression have been developed using the SIMOA method (Figure 1A), which is an ultrasensitive ELISA technology with a detection limit in the $\mathrm{fg}^{-1}$ range 
(Rissin et al., 2010). After a series of assay optimizations, we achieved the best working performance with an assay limit of detection (LOD) of $0.0157 \mathrm{pg} \mathrm{mL}^{-1}$ and limit of quantitation of $0.068 \mathrm{pg} \mathrm{mL}^{-1}$. This represents a nearly 3,000 -fold increase of sensitivity compared with that of the commercially available $\mathrm{N}$ protein ELISA kit. Therefore, this permitted us to measure viral antigen levels directly in patients' blood.

Thereafter, all plasma samples were analyzed, and the findings were compared with the qPCR results of pharyngeal swabs. Patients with COVID-19 and positive or negative qPCR results for SARS-CoV-2 RNA using pharyngeal swabs were classified into the SARS-CoV-2-positive or SARS-CoV-2-negative group, respectively. The mean average enzymes per bead (AEB) for plasma samples was 0.003 ( $95 \%$ confidence interval $[\mathrm{CI}]=0.002-0.004)$ for healthy controls $(n=27)$, versus $0.012(95 \% \mathrm{CI}=0.003-0.022)$ for the SARS-CoV-2-negative group ( $n=50$, Figure 1B). Nevertheless, the mean AEB for plasma samples was $0.078(95 \%$ $\mathrm{CI}=0.012-0.145)$ in the SARS-CoV-2-positive group $(n=39$, Figure 1B). Accordingly, the corresponding circulating $\mathrm{N}$ protein levels averaged $0.02 \mathrm{pg} \mathrm{mL}^{-1}(95 \% \mathrm{CI}=-0.02-0.06)$ in the healthy control group, $0.86 \mathrm{pg} \mathrm{mL}^{-1}(95 \% \mathrm{CI}=-0.18-$ $1.91)$ in the SARS-CoV-2-negative group, and $9.47 \mathrm{pg} \mathrm{mL}^{-1}$ (95\% CI=1.03-17.9) in the SARS-CoV-2-positive group (Figure 1C). Of 39 plasma samples obtained from patients in the SARS-CoV-2-positive group, circulating $\mathrm{N}$ protein was detected in 29 samples $(74.4 \%)$. By contrast, among 50 plasma samples in the SARS-CoV-2-negative group, $\mathrm{N}$ antigen was detected in 16 specimens $(32.2 \%)$. Thus, the detection rate of circulating $\mathrm{N}$ protein was 2.3 -fold higher in the SARS-CoV-2-positive group than in the SARS-CoV-2-negative group $(74.4 \%$ vs. $32.0 \%, P=0.0001)$.

Finally, the kinetics of circulating $\mathrm{N}$ antigen was compared with the qPCR results for pharyngeal swabs in individual patients. Among 10 patients with severe or critically severe illness, circulating $\mathrm{N}$ protein was detected in six patients with SARS-CoV-2 RNA positivity and one patient with susceptible virus present in pharyngeal swabs (Figure 1D). Interestingly, $\mathrm{N}$ antigen was also identified in two patients with severe COVID-19 despite SARS-CoV-2 RNA negativity in pharyngeal swabs. N antigen was not detected in one SARSCoV-2-positive patient. In three of four patients who died of COVID-19, circulating $\mathrm{N}$ antigen levels were either high or maintained at different time points (Figure 1E).

Concerning 25 patients with mild COVID-19, N antigen was detected in the blood of 13 patients with positive qPCR results for SARS-CoV-2 in pharyngeal swabs (Figure 1F). N antigen in the blood was detected in five patients with negative SARS-CoV-2 RNA results for pharyngeal swabs. N protein was not detected in seven patients with positive qPCR results for SARS-CoV-2.

Concerning the main findings of the study, (1) circulating $\mathrm{N}$ protein could be quantified at the $\mathrm{pg} \mathrm{mL}^{-1}$ level via the
SIMOA method, and its levels accurately discriminated healthy controls from patients with COVID-19. (2) Compared with positive results for SARS-CoV-2 in respiratory samples using qPCR, the detection rate of $\mathrm{N}$ antigen was $74.4 \%$ in plasma samples. (3) Circulating $\mathrm{N}$ antigen levels were high or sustained in three of four patients who died of COVID-19, suggesting the presence of $\mathrm{N}$ antigen in the blood might be a potential risk factor, which is worthy of further investigation.

Accumulating evidence indicates that the respiratory system is the main target of SARS-CoV-2. When dissecting the causes of mortality in 85 patients, respiratory failure, shock and ARDS were identified in $94 \%, 81 \%$, and $74 \%$ of cases, respectively (Huang et al., 2020). In the study, we also used the SIMOA method to detect the $\mathrm{N}$ protein in pharyngeal swabs. However, no significant difference of circulating $\mathrm{N}$ protein levels was observed between SARS-CoV-2-negative and SARS-CoV-2-positive swabs according to qPCR $(P>0.10)$. One possible reason could be that we did not use detergent to degrade the cell membrane for $\mathrm{N}$ antigen release. Alternatively, the viral load could be below the LOD in these samples.

Because of the low performance of current technology (Lippi et al., 2020), it remains unclear whether SARS-CoV-2 could circulate in the blood and infect other organs for further destruction. In clinical practice, inconsistent results have been obtained using qPCR for SARS-CoV-2 in RNA extracted from respiratory samples because of the different methods of specimen collection, RNA quality after extraction, and sensitivity of qPCR amplification (Yuan et al., 2020). Thus, quantification of antigen levels in the blood could supplement qPCR assays for diagnosis and improve our understanding of disease progression. Compared with qPCR results as the standard criteria, the SIMOA method achieved a detection rate of $74.4 \%$ for $\mathrm{N}$ antigen in the blood in patients with COVID-19. To the best of our knowledge, this is by far the highest detection rate reported. Moreover, circulating $\mathrm{N}$ protein detected using the SIMOA method discriminated patients with COVID-19 from healthy controls. In our study, circulating $\mathrm{N}$ antigen was also detected in patients with COVID-19 whose qPCR results for pharyngeal swabs were negative, supporting the added value of $\mathrm{N}$ antigen measurements in the blood for disease diagnosis and severity assessments. However, some limitations of this study deserve mention. First, we could not clarify the entire time course of blood samples in some patients. Second, the qPCR results of swabs indicated the presence of susceptible virus in several specimens. Finally, each run of the assay required $2 \mathrm{~h}$, indicating that further optimization is needed.

In summary, using SIMOA technology, $\mathrm{N}$ antigen levels in circulation were detected in $74.4 \%$ of plasma samples from patients with COVID-19 compared with the qPCR results. Blood antigen quantification could serve as a supplement to 
A

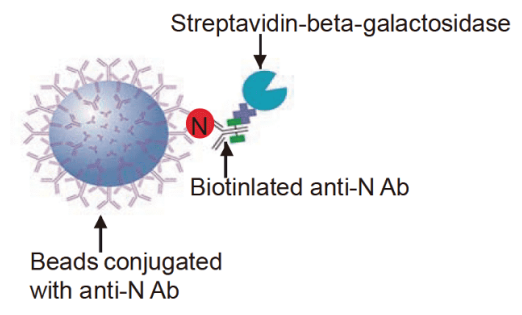

B

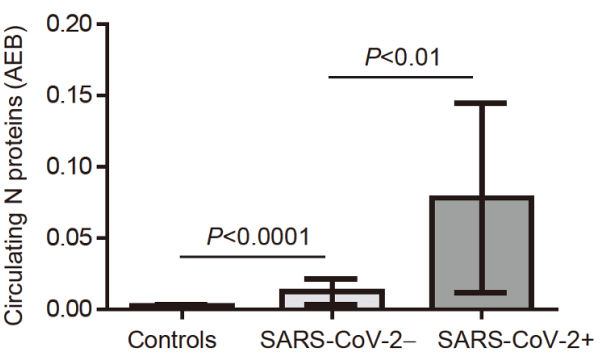

C

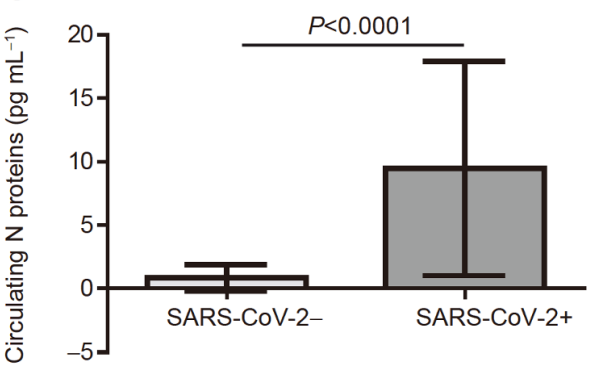

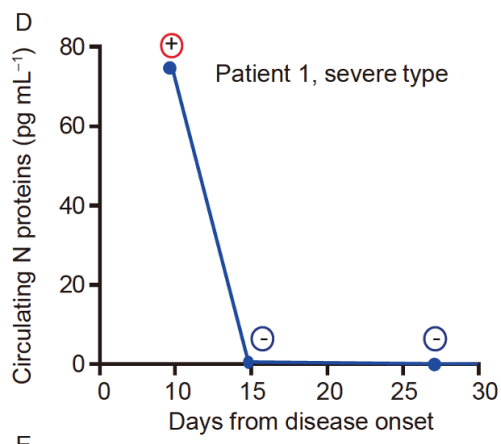
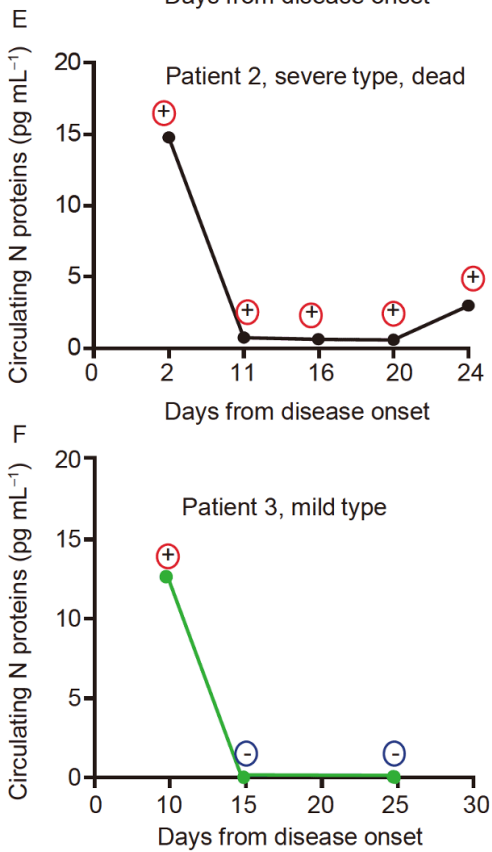

Figure 1 Single molecular array (SIMOA) analysis of nucleocapsid protein (N protein) in the blood of patients with coronavirus disease 2019 (COVID-19). A, Mechanisms of SIMOA for N protein detection. B, The number of average enzymes per bead (AEB), representing a parameter for $\mathrm{N}$ protein quantification, was calculated using plasma samples from healthy controls and patients with COVID-19 for whom qPCR assays for SARS-CoV-2 using pharyngeal swabs were positive or negative. C, Plasma levels of the N protein in patients with COVID-19 who had positive or negative qPCR results for SARS-CoV-2 using pharyngeal swabs. Data are expressed as the mean with $95 \%$ confidence intervals. D and $\mathrm{E}$, The kinetics of circulating $\mathrm{N}$ protein in patients with severe COVID-19. F, The kinetics of circulating N protein in one patient with mild COVID-19. Positive and negative qPCR assays for SARS-CoV-2 in pharyngeal swabs are indicated by red "plus" and blue "minus" symbols, respectively.

qPCR for disease diagnosis, especially for SARS-CoV-2negative respiratory samples according to $\mathrm{qPCR}$.

Compliance and ethics The author(s) declare that they have no conflict of interest. This study and all the relevant experiments were approved by the Beijing Youan Hospital Research Ethics Committee, and written informed consent was obtained from each participant in accordance with the Declaration of Helsinki.

Acknowledgements The authors gratefully appreciated the participation of patients in this study. We thank Bing Xu and Junhui Li for their excellent assistance for sample preparation and data processing. This work was supported by the Ministry of Science and Technology of the People's Republic of China (2020YFC0844900, 2020YFC0841700, and 2020YFC0848700), the National Natural Science Foundation of China (81470566, 81670765, 81772165, and 81974303), the China Primary Health Care Foundation-Youan Medical Development Fund (BJYAYY2020PY-01) and the funds from Neoline, Gbio and Quanterix. The funders had no role in study design, data collection and analysis, decision to publish, or preparation of the manuscript.

\section{References}

Bavishi, C., Bonow, R.O., Trivedi, V., Abbott, J.D., Messerli, F.H., and Bhatt, D.L. (2020). Acute myocardial injury in patients hospitalized with COVID-19 infection: A review. Prog Cardiovasc Dis https://doi. org/10.1016/j.pcad.2020.05.013.

Gabarre, P., Dumas, G., Dupont, T., Darmon, M., Azoulay, E., and Zafrani, L. (2020). Acute kidney injury in critically ill patients with COVID-19. Intens Care Med 46, 1339-1348.

Huang, C., Wang, Y., Li, X., Ren, L., Zhao, J., Hu, Y., Zhang, L., Fan, G., $\mathrm{Xu}, \mathrm{J} ., \mathrm{Gu}, \mathrm{X}$., et al. (2020). Clinical features of patients infected with 2019 novel coronavirus in Wuhan, China. Lancet 395, 497-506.

Lippi, G., Mattiuzzi, C., Bovo, C., and Plebani, M. (2020). Current laboratory diagnostics of coronavirus disease 2019 (COVID-19). Acta Biomed 91, 137-145.

Mak, G.C., Cheng, P.K., Lau, S.S., Wong, K.K., Lau, C.S., Lam, E.T., Chan, R.C., and Tsang, D.N. (2020). Evaluation of rapid antigen test for detection of SARS-CoV-2 virus. J Clin Virol 129, 104500.

Phipps, M.M., Barraza, L.H., LaSota, E.D., Sobieszczyk, M.E., Pereira, M. R., Zheng, E.X., Fox, A.N., Zucker, J., and Verna, E.C. (2020). Acute liver injury in COVID-19: prevalence and association with clinical 
outcomes in a large U.S. cohort. Hepatology 72, 807-817.

Rissin, D.M., and Walt, D.R. (2006). Digital concentration readout of single enzyme molecules using femtoliter arrays and poisson statistics. Nano Lett 6, 520-523.

Rissin, D.M., Kan, C.W., Campbell, T.G., Howes, S.C., Fournier, D.R., Song, L., Piech, T., Patel, P.P., Chang, L., Rivnak, A.J., et al. (2010). Single-molecule enzyme-linked immunosorbent assay detects serum proteins at subfemtomolar concentrations. Nat Biotechnol 28, 595-
599.

Scohy, A., Anantharajah, A., Bodéus, M., Kabamba-Mukadi, B., Verroken, A., and Rodriguez-Villalobos, H. (2020). Low performance of rapid antigen detection test as frontline testing for COVID-19 diagnosis. J Clin Virol 129, 104455.

Yuan, C., Zhu, H., Yang, Y., Cai, X., Xiang, F., Wu, H., Yao, C., Xiang, Y., and Xiao, H. (2020). Viral loads in throat and anal swabs in children infected with SARS-CoV-2. Emerg Microb Infect 9, 1233-1237.

\section{SUPPORTING INFORMATION}

The supporting information is available online at https://doi.org/10.1007/s11427-020-1830-8. The supporting materials are published as submitted, without typesetting or editing. The responsibility for scientific accuracy and content remains entirely with the authors. 